CORRECTION

https://doi.org/10.1038/s41586-018-0584-2

\title{
Publisher Correction: The NORAD lncRNA assembles a topoisomerase complex critical for genome stability
}

Mathias Munschauer, Celina T. Nguyen, Klara Sirokman, Christina R. Hartigan, Larson Hogstrom, Jesse M. Engreitz, Jacob C. Ulirsch, Charles P. Fulco, Vidya Subramanian, Jenny Chen, Monica Schenone, Mitchell Guttman, Steven A. Carr \& Eric S. Lander

Correction to: Nature https://doi.org/10.1038/s41586-018-0453-z, published online 27 August 2018.

In the 'Reviewer information' section of this Letter, 'S. Diederichs' was incorrectly listed as 'S. Diedrichs'; this has been corrected online. 